Meta

Journal des tradlucteurs

Translators' Journal

\title{
Les textes traduits et leur utilisation en teminologie
}

\section{Robert Dubuc}

Volume 19, numéro 4, décembre 1974

URI : https://id.erudit.org/iderudit/002632ar

DOI : https://doi.org/10.7202/002632ar

Aller au sommaire du numéro

Éditeur(s)

Les Presses de l'Université de Montréal

ISSN

0026-0452 (imprimé)

1492-1421 (numérique)

Découvrir la revue

Citer cet article

Dubuc, R. (1974). Les textes traduits et leur utilisation en teminologie. Meta, 19(4), 205-208. https://doi.org/10.7202/002632ar d'utilisation que vous pouvez consulter en ligne.

https://apropos.erudit.org/fr/usagers/politique-dutilisation/ 


\section{LES TEXTES TRADUITS}

\section{ET LEUR UTILISATION EN TERMINOLOGIE}

Les services de terminologie liés à des bureaux de traduction ont la tentation constante de procéder au dépouillement systématique des traductions de la maison puis de là de passer au dépouillement de toutes les traductions dont la tenue semble bonne.

Les avantages du procédé semblent évidents à première vue. Il peut permettre d'unifier la terminologie à l'intérieur d'un service. Le coût de la recherche est ramené à un prix minimal : n'importe quel bon bilingue peut retrouver les équivalents entre deux textes traduits l'un de l'autre et à un rythme qui défie toute comparaison avec celui des recherches dans les textes originaux.

Devant ces avantages non négligeables, il nous est apparu nécessaire d'étudier de plus près quelques exemples de terminologie de traduction, afin d'éclairer ou de nuancer le principe énoncé au Congrès de terminologie de Genève : «L'information terminologique doit toujours être tirée de textes originaux dignes de foi. »

Les textes que nous avons étudiés sont de nature technique; ils touchent, le premier au domaine de la banque, le deuxième aux télécommunications et le troisième à la statistique. Cette étude a été rendue possible grâce à la collaboration des étudiants stagiaires en terminologie ${ }^{1}$ et de M. Jean Hesse, ex-directeur de la terminologie aux Nations unies.

Pour le vocabuloire bancaire, c'est le rapport annuel du Fonds monétaire international ${ }^{2}$ dans ses versions anglaise et française qui a servi de base au relevé terminologique. Ce dépouillement a permis d'établir rapidement 251 fiches bilingues complètes. Le dépouillement s'est fait de façon plutôt exhaustive que sélective. Les données qui suivent ont été établies d'après un échantillonnage au hasard et extrapolées à l'ensemble de la recherche.

$32 \%$ des fiches posaient des problèmes difficiles de terminologie et proposaient des solutions acceptables.

Par contre, $32 \%$ proposaient des solutions déjà connues et faciles à obtenir.

1. Pour les télécommunications, Mlles Danielle Lagueux et Viviane Forest; pour la statistique, Mmes Andrée Mayers et Louise Letendre (été 1974).

2. International Monetary Fund, Annual Report, Washington (D.C.), 1968, 200 p. Fonds monétaire international, Rapport annuel, Washington (D.C.), 1968, 196 p. 
$44 \%$ des fiches représentaient de la terminologie maison, non susceptible d'application hors du Fonds monétaire international.

$20 \%$ des fiches semblaient en contradiction avec l'usage établi dans ce domaine ou encore de simples calques des expressions anglaises, sans référence à la réalité de la langue d'arrivée ${ }^{3}$.

Le deuxième travail de dépouillement a porté sur un extrait des actes de la $\mathrm{XII}^{e}$ assemblée plénière du Comité consultatif international des radiocommunications ${ }^{4}$. Ce travail a donné lieu à quelque 400 fiches bilingues à double contexte. L'aspect étudié - les satellites de télécommunication - présente une indigence assez grande de documents originaux en français. La traduction de ces actes semble bien documentée. Le nombre de problèmes terminologiques importants posés et résolus de façon satisfaisante s'élève à près de $85 \%$ des expressions relevées. Ce qui est cependant instructif, c'est la typologie des fiches non retenues ${ }^{5}$.

Il y a d'abord les fiches nettement erronées ou douteuses (un petit nombre) parmi lesquelles on trouve, par exemple, transmission delay rendu par temps de propagation au lieu de retard de propagation ou de diffusion; signal attenuation rendu par évanouissement du signal (l'évanouissement est en général total et non une simple atténuation) ; l'adoption de brouillage comme équivalent d'interference, ce qui laisse jamming sans équivalent spécifique. Or, le brouillage est en général volontaire et l'interference accidentel. Enfin, dipole rendu par doublet apparaît comme un néologisme d'assez bonne frappe mais sans sanction de l'usage.

Mais la caractéristique la plus fréquente vient de la tendance à la surcaractérisation de la terminologie utilisée, signe infaillible de l'influence de la traduction. On cherche en fait à en dire trop par crainte de ne pas en dire assez. Voici quelques exemples : Discontinuity : discontinuité de temps, Exhaust Type Plasma : plasma dû aux échappements, Noise-Like Interference : brouillage à caractéristique de bruit, Pay-Load Limitation : limitation imposée aux charges utiles, Frequency-Reversed System : système avec inversion des bandes de fréquences, Unused Satellite : satellite non en cours de fonctionnement. Tous ces équivalents sont inutilement lourds et grèvent la terminologie de la langue d'arrivée d'expressions non maniables. Des expressions comme plasma d'échappement, brouillagebruit, limitation des charges utiles, système avec inversion des fréquences, satellite non utilisé, moins précises peut-être, auraient toutefois allégé la terminologie. Une appellation ne doit pas être une définition.

Cette analyse a permis de mettre en évidence une certaine incohérence dans l'utilisation des équivalents d'un même terme, ainsi phase lock est rendu tantôt

3. Voici quelques exemples : articles of agreement : statuts; executive director : administrateur; governor (of the Bank) : gouverneur; reserve position : position de réserve (situation des réserves); rules and regulations : règles et règlements (surtraduction); surplus country : pays excédentaire; terms of trade : termes d'échange (pour conditions).

4. Comité consultatif international des radiocommunications, XIIe Assemblée plénière, Genève, Union internationale des télécommunications, 1970 , vol. 4, partie $2,465 \mathrm{p}$.

International Radio Consultative Committee, XIIth Plenary Assembly, Geneva, 1970, Vol. 4, Part 2, 466 p.

5. L'analyse de ce travail s'est faite par voie d'échantillonnage et d'extrapolation, comme pour le travail précédent. 
par asservissement de phase, tantôt par blocage de phase, unphased tantôt par non en phase, tantôt par à répartition aléatoire.

Enfin, la troisième étude a consisté à comparer la terminologie statistique utilisée dans une traduction ${ }^{6}$ avec les données d'une recherche terminologique originale. Sur 137 expressions recensées, on ne note que 27 cas de concordance complète, ce qui laisse un solde de 110 cas de disparité. Comment expliquer un tel écart?

Il semble bien que les traducteurs ont voulu mettre au point « leur » terminologie, sans tenir compte d'un usage auquel ils ne faisaient visiblement pas confiance. Même les travaux de normalisation de l'AFNOR ont été entièrement ignorés. Il en résulte une terminologie qui s'écarte sans raison de la terminologie courante, parce que les auteurs ont cru devoir surcaractériser les expressions ou mettre au point des expressions nouvelles par crainte exagérée d'être trop près de l'anglais. Certains exemples de surcaractérisation : courbe en forme de cloche pour courbe en cloche, courbe de densité normale pour courbe normale, courbe de densité en $\mathrm{J}$, pour courbe en $\mathrm{J}$, intervalle entre les $10^{\mathrm{e}}$ et $90^{\mathrm{e}}$ centiles pour intervalle décile. Pourtant, les expressions que les auteurs se sont refusé à employer sont courantes et difficilement répréhensibles.

Les auteurs ont aussi craint souvent de rendre distribution par distribution, curve par courbe et frequency par fréquence. «Ces scrupules font voir trop de délicatesse », l'usage ayant largement ratifié ces équivalences. On s'explique en outre difficilement que les traducteurs aient ignoré coefficient de pondération pour weight, et aient préféré ajustement d'une courbe à lissage d'une courbe, les deux expressions rejetées étant largement répandues.

\section{Conclusion}

De ces trois expériences, il faut conclure à la sagesse du principe adopté au Congrès de terminologie de Genève, parce que les textes traduits sont effectivement dangereux. Dans les meilleures conditions, la terminologie y souffre d'une certaine insécurité qui risque de la gauchir et de mener à plus ou moins brève échéance à une terminologie de traduction en marge de la langue vivante.

Le trait le plus frappant de la terminologie des textes traduits est bien cette tendance à surcaractériser les expressions. Ce procédé finit par alourdir indûment la terminologie de la langue d'arrivée au point de la rendre difficilement maniable.

La seconde caractéristique vient des écarts aux termes usuels, particulièrement sensibles dans la première et la troisième étude. Ces disparités d'avec l'usage finissent par créer une véritable langue de traduction, quand ce n'est pas une langue de traducteurs. Même si on ne peut ériger en dogme le principe de la primauté de l'usage, il reste qu'on peut difficilement faire de la bonne terminologie en en faisant abstraction.

6. Spiegel, Murray R., Théorie et applications de la statistique, New York, McGraw-Hill International, "Série Schaum», 1972, 358 p. (traduction de A. Ergas et J.-F. Marcotorchino). 
À cet égard, les économies réalisées par le dépouillement des traductions risquent d'accentuer le dédale des terminologies et finiront par coûter, à cause des risques de confusion, beaucoup plus cher que la terminologie faite sérieusement.

L'expérience du texte des télécommunications, de beaucoup la plus positive, montre qu'il y a moyen de tirer d'une traduction une information terminologique valable, mais elle nous incite à la plus grande prudence, encore une fois, si l'on ne veut pas perdre le contact avec la réalité.

ROBERT DUbuC 